Präv Gesundheitsf 2021 · 16:144-149 https://doi.org/10.1007/s11553-020-00804-x Eingegangen: 29. April 2020

Angenommen: 25. Juni 2020

Online publiziert: 21. Juli 2020

๑) Der/die Autor(en) 2020

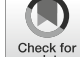

Stephanie Karg (D) - Beate Blättner · Talih Süleyman Igde

Hochschule Fulda, Fulda, Deutschland

\section{Gesundheitsförderung mit Geflüchteten}

\section{Bedarf aus Sicht der Betroffenen}

\begin{abstract}
Geflüchtete gehören zu einer sozial wie gesundheitlich besonders benachteiligten Bevölkerungsgruppe und stellen deswegen eine wichtige Adressatengruppe für Gesundheitsförderung dar. In Deutschland existieren einige vielversprechende und z. T. stark partizipative Projekte, die u. a. auf der Datenbank des Kooperationsverbundes Gesundheitliche Chancengleichheit zu finden sind. Dennoch ist die Gesundheitsförderung mit Geflüchteten in Deutschland insgesamt wenig entwickelt und noch vergleichsweise wenig an den konzeptionellen Ideen der Gesundheitsförderung in Lebenslagen ausgerichtet.
\end{abstract}

\section{Hintergrund und Fragestellung}

Ende des Jahres 2018 befanden sich weltweit 70,8 Mio. Menschen auf der Flucht. 25,9 Mio. davon flohen vor Konflikten, Verfolgung oder schweren Menschenrechtsverletzungen. Über die Hälfte kommen aus den drei Ländern Syrien, Afghanistan und Südsudan [11]. Die Genfer Flüchtlingskonvention (Art. 1 GFK) definiert Geflüchtete als Personen, die sich aus begründeter Furcht vor Verfolgung wegen ihrer Rasse, Religion, Nationalität, politischen Überzeugung oder Zugehörigkeit zu einer bestimmten sozialen Gruppe außerhalb des Landes befinden, dessen Staatsangehörigkeit sie besitzen oder in dem sie als Staatenlose ihren vorherigen gewöhnlichen Aufenthalt hatten und dessen Schutz sie nicht in Anspruch nehmen können oder aufgrund der Furcht nicht wollen.
Die meisten Geflüchteten leben in den Nachbarländern ihrer Heimatstaaten, in der Regel Länder mit niedrigem oder mittlerem Einkommen. In Deutschland gab es Ende des Jahres 2018 insgesamt 1,1 Mio. Geflüchtete [11]; Deutschland war damit das fünftgrößte Aufnahmeland nach der Türkei, Pakistan, Uganda und dem Sudan. Im Jahr 2016 wurden während der sog. Flüchtlingskrise in Deutschland 745.545 Asylanträge gestellt, davon 722.370 Erstanträge. Im Jahr 2019 waren es aufgrund einer strikteren Politik der Grenzschließung nach Europa 165.938, davon 142.509 Erstanträge. Die drei häufigsten Herkunftsländer waren im Januar 2020 Syrien, Irak und Afghanistan [3].

Weniger als die Hälfte der Asylbewerberinnen und Asylbewerber hat die Chance, zumindest zeitlich befristet in Deutschland zu bleiben. In 29,4\% der in 2019 entschiedenen Fälle erfolgte eine Ablehnung des Antrages auf Asyl, in 32,4\% der Fälle eine formelle Entscheidung aufgrund der Zuständigkeit eines anderen EU-Landes. In 24,5\% wurde die Rechtsstellung als Flüchtling ausgesprochen, in 10,6\% ein subsidiärer Schutz gem. $\$ 4$ Abs. 1 des Asylgesetzes (AsylG) und in 3,2\% ein Abschiebungsverbot gem. $₫ 60$ Abs. 5 oder 7 Aufenthaltsgesetzes (AufenthG; [3]). Flüchtlingsschutz ( $\$ 3$ Abs. 1 AsylVfG) und Asylberechtigung aufgrund politischer Verfolgung (Art. 16 a Abs. 1 GG) führen zu einem Anspruch auf einen 3-jährigen Aufenthalt. Fallen die Gründe für die Anerkennung in dieser Zeit nicht weg, wird eine Niederlassung erlaubt. Subsidiär Schutzberechtigten wird die Aufenthaltserlaubnis für ein Jahr erteilt, eine
Verlängerung um zwei weitere Jahre ist möglich. Wenn ein Abschiebungsverbot vorliegt, soll eine Aufenthaltserlaubnis für mindestens ein Jahr erfolgen. Diese kann nach $\$ 60$ Abs. 7 AufenthG auch erteilt werden, wenn der geflüchteten Person aufgrund einer lebensbedrohlichen oder schwerwiegenden Erkrankung nach ihrer Rückkehr die Gefahr einer wesentlichen Gesundheitsverschlechterung droht. Dafür muss eine nachvollziehbare Diagnose vorliegen, aus dem sich die ernsthafte Gefahr ergibt [2]. • Abb. 1 gibt einen Überblick über den möglichen Ausgang eines Asylverfahrens.

Erfahrungen, die die Flucht veranlasst haben und Erfahrungen während der Fluchtkönnen lebensbedrohlich gewesen sein und mit massiven Gesundheitsgefährdungen körperlicher und seelischer Art einhergehen [6]. Geflüchtete stellen entsprechend gesundheitlich eine besonders vulnerable Personengruppe dar, die zugleich nur beschränkten Zugang zur medizinischen Versorgung und zu präventiven Maßnahmen hat. Welche gesundheitlichen Leistungen Geflüchtete im Bundesgebiet erhalten, ist im Asylbewerberleistungsgesetz ( $\$ 4$ und $\$ 6$ AsylbLG) geregelt. Dort ist die Gesundheitsversorgung auf die Behandlung von akuten Erkrankungen, die Versorgung und Unterstützung bei Schwangerschaft und Geburt sowie Schutzimpfungen und Vorsorgeuntersuchungen beschränkt. Weitere Leistungen können gewährt werden, wenn sie im Einzelfall zur Sicherung der Gesundheit unerlässlich sind. Geflüchtete, die eine Aufenthaltserlaubnis ( $\$ 24$ Abs. 1 AufenthG) besitzen und die beispielsweise Folter, Vergewaltigung oder sonstige schwere Formen psychi- 


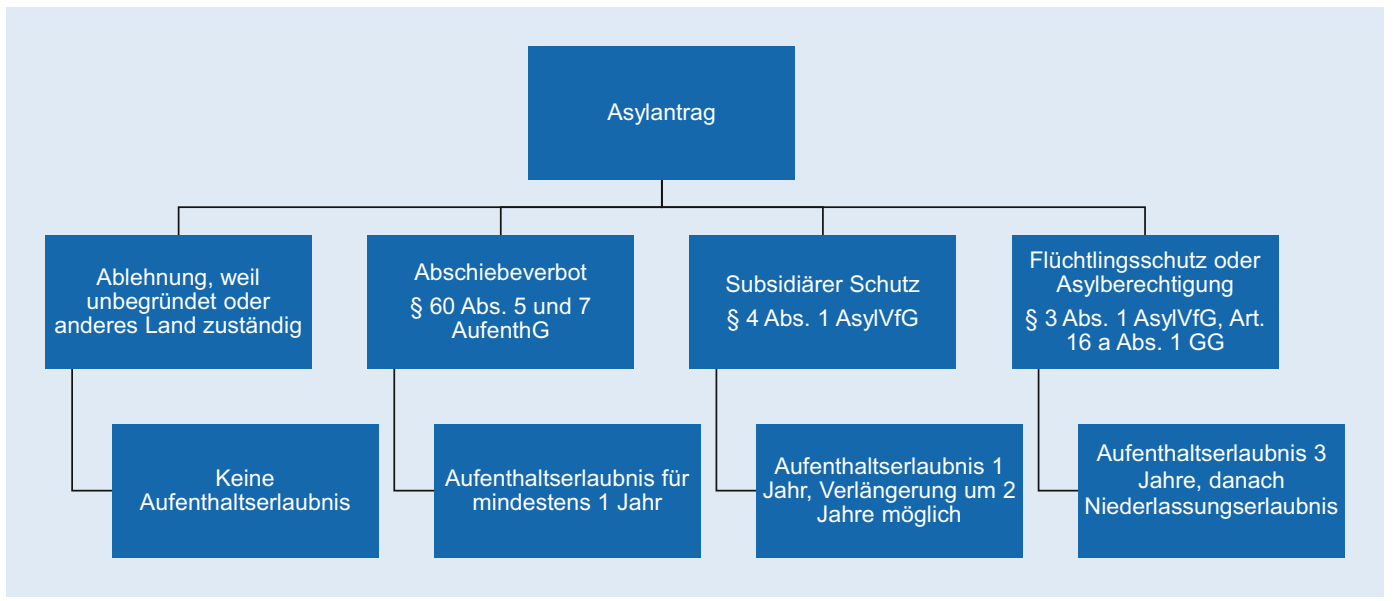

Abb. $1 \triangleleft$ Möglicher Ausgang eines Asylverfahrens (nach [2]) scher, physischer oder sexueller Gewalt erlitten haben, wird die erforderliche medizinische Hilfe gewährt. Maßnahmen der Gesundheitsförderung und der nichtmedizinischen Prävention im Sinne des Sozialgesetzbuches V (SGB V) sind allerdings nicht vorgesehen.

Da sich Gesundheitsförderung seit der Erklärung von Alma-Ata 1978, der internationalen Konferenz über primäre Gesundheitsversorgung, die Verringerung gesundheitlicher Ungleichheit zum Ziel setzt [8], sind Geflüchtete damit eine nicht hinreichend mit Interventionen der Gesundheitsförderung versorgte Bevölkerungsgruppe.

Seit der Jakarta-Erklärung zur Gesundheitsförderung für das 21. Jahrhundert (1997) gilt Gesundheitsförderung international als ein Prozess, der Menschen befähigen soll, mehr Kontrolle über ihre Gesundheit zu erlangen und sie durch Beeinflussung der Determinanten für Gesundheit zu verbessern [8]. Dahlgren und Whitehead [4] benennen als solche Determinanten auf der Makroebene Umweltbedingungen wie Wohnen, Landwirtschaft und Lebensmittelproduktion, Bildung, Arbeitsumfeld. Arbeitslosigkeit, Wasser und Hygiene oder Gesundheitswesen, auf der Mesoebene die Lebensweise und auf der Mikroebene schließlich Alter, Geschlecht und erbliche Faktoren. Im sozioökologischen Modell der Gesundheit wird die Reziprozität dieser Determinanten als zentral betrachtet. Für die Gesundheitsförderung mit Geflüchteten bedeutet dies konkret, Lebensstilthemen wie Ernährung und Bewegung nicht ohne den Blick auf die Chancen Geflüchteter auf Selbstbestimmung in ihren Lebensbedingungen zu richten.

Entsprechend dem partizipativen Ansatz der Gesundheitsförderung interessierte zunächst die Sicht in Deutschland lebender Geflüchteter auf ihre Erfahrungen und Bedürfnisse. Dabei war dieser weite Ansatz der Determinanten von Gesundheit leitend.

\section{Studiendesign und Unter- suchungsmethoden}

Die Sichtweisen Geflüchteter wurden in Einzel- und Gruppengesprächen von Geflüchteten erhoben, die in Gemeinschaftsunterkünften oder eigenen Wohnungen lebten. Dafür wurde nach dem Prinzip des Sammelns, Prüfens, Sortierens und Subsumierens [7] ein Leitfaden entwickelt, der sich in die drei Themengruppen Lebensumstände, Gesundheit und Fluchterfahrungen gliedert. Jeder der Themenbereiche beginnt mit einer Erzählaufforderung. So beginnt der erste Themenbereich mit der Aufforderung „Bitte erzählen Sie uns wie Sie Ihren Alltag gestalten“, gefolgt von Nachfragen zum Tagesablauf, dem sozialen Umfeld, Arbeit oder Freizeitgestaltung, wenn diese Aspekte nicht erwähnt wurden. Ergänzend konnten Aufrechterhaltungs- und Steuerungsfragen folgen sowie konkrete Ermutigungen, bestimmte Situationen ausführlicher zu erzählen.

Insgesamt wurden 13 Interviews mit 6 Familien, 3 Männern und 4 Frauen geführt, in Summe 30 Personen inklusive Kindern. Darunter lebten 3 Familien mit 12 Personen in eigenen Wohnungen und insgesamt 18 Personen in Gemeinschaftsunterkünften. Die Interviews mit geflüchteten Personen wurden grundsätzlich mit einer weiblichen und einer männlichen Interviewperson geführt unter Anwesenheit einer Person einer Wohlfahrtsorganisation, die den Feldzugang ermöglichte und wenn notwendig einem Sprachmittler aus der Wohlfahrtsorganisation. Zum Teil fungierten Familienmitglieder als Sprachmittler. Bei den Interviewenden handelt es sich um dafür geschulte Studierende. Die Gruppeninterviews wurden digital aufgezeichnet, zusätzlich wurde ein Erinnerungsprotokoll zum Ablauf und Inhalt des Gruppengesprächs verfasst. Die Durchführung der Interviews erfolgte im Zeitraum von Februar bis Juli 2019 mit einer Gesprächsdauer von 10 bis $60 \mathrm{~min}$, je nach Erzählbereitschaft und abhängig von den vorhandenen Sprachkenntnissen. - Tab. 1 gibt einen Überblick über das erhobene Datenmaterial.

Die Transkription der Gesprächsaufnahmen erfolgte im deutschen bzw. ins Deutsche übersetzten Text wörtlich [5], Aussagen in der Originalsprache wurden nicht transkribiert. Die Analyse der Daten erfolgte dreischrittig durch offenes, axiales und selektives Kodieren. Dabei wurden die empirischen Daten zunächst in Konzepte und schließlich in Kategorien überführt [10]. 
Präv Gesundheitsf 2021 • 16:144-149 https://doi.org/10.1007/s11553-020-00804-x

(c) Der/die Autor(en) 2020

\section{S. Karg • B. Blättner · T. Süleyman Igde}

\section{Gesundheitsförderung mit Geflüchteten. Bedarf aus Sicht der Betroffenen}

\section{Zusammenfassung}

Hintergrund. Weltweit sind Millionen von Menschen aufgrund von Verfolgung, politischer Diskriminierung oder Krieg auf der Flucht. Geflüchtete gehören zu einer vulnerablen und gesundheitlich benachteiligten Bevölkerungsgruppe, die in Deutschland zugleich nur beschränkten Zugang zur medizinischen Versorgung und zu präventiven Maßnahmen hat. Sie bedarf besonderer Aufmerksamkeit in der Gesundheitsförderung.

Ziel der Arbeit. Es interessierte die Sicht in Deutschland lebender Geflüchteter auf ihre Erfahrungen und Bedürfnisse.

Methoden. Es wurden 13 leitfadengestützte Interviews mit insgesamt 30 Geflüchteten aus Afghanistan, Irak, Somalia und Syrien geführt, die entweder in Gemeinschaftsunterkünften oder in eigenen Wohnungen leben. Interviewt wurden Familien im Gruppeninterview sowie einzeln Männer und Frauen zu ihren Erfahrungen im Alltag und auf der Flucht sowie zum Thema Gesundheit.

Ergebnisse. Geflüchtete haben viel investiert, um nach Deutschland zu gelangen und investieren z. T. viele Ressourcen, um ihrem Ziel der Selbstbestimmung über ihr Leben ein Stück näher zu kommen. Ihr Aufenthaltsstatus, nicht anerkannte Qualifikationen und fehlende Arbeitschancen, die Wahrnehmung von Misstrauen, Willkür und Ausgrenzung, Sprachbarrieren, Gesundheitsbeeinträchtigungen und ein eingeschränkter Zugang zum
Gesundheitssystem erschweren das Erreichen der Ziele.

Schlussfolgerung. Durch den Verlust von Selbstbestimmung und Mangel an Erfahrungen zur Teilhabe an sozial anerkannten Aktivitäten im Ankunftsland kann es zu einer Abnahme an Gesundheitsressourcen kommen. Daher bedarf es einer stetigen Unterstützung der Geflüchteten, das Empfinden von Kohärenz zu erhalten oder zu entwickeln.

Schlüsselwörter

Geflüchtete - Gesundheitsressourcen . Persönliche Autonomie - Kohärenzgefühl · Lebensqualität

\section{Health promotion for refugees. Needs from the perspective of people concerned}

\section{Abstract}

Background. Millions of people worldwide are on the run due to persecution, political discrimination or war. Refugees belong to a vulnerable and health-disadvantaged population group, which in Germany has at the same time only limited access to medical care and preventive measures. Thus, they need special attention in health promotion. Objectives. Refugees living in Germany were interviewed about their experiences and needs.

Methods. In all, 13 guideline-based interviews were conducted with a total of 30 refugees from Afghanistan, Iraq, Somalia and Syria, who either live in refugee accommodations or in their own apartments. Families were interviewed in a group interview as well as men and women individually on their experiences in everyday life and while they were fleeing their country of origin and on the subject of health.

Results. Refugees have invested significant resources to get to Germany and are investing a lot of resources in order to get closer to their goal of self-determination over their lives. Their residence status, unrecognized qualifications and lack of job opportunities, the perception of mistrust, arbitrariness and exclusion, language barriers, health impairments and restricted access to the health system make it difficult to achieve their goals.

Conclusions. Due to the loss of selfdetermination and a lack of experience in participating in socially recognized activities in the new country where they have settled, there may be a decrease in health resources. Therefore, there is a need for constant support to help refugees to maintain or develop a sense of coherence.

Keywords

Refugees · Health resources - Personal autonomy · Sense of coherence · Quality of life

\section{Ergebnisse}

\section{Ressourcen}

Ich bin alleine erst mal gekommen, ganz alleine. Von Irak nach Türkei gelaufen, Türkei nach Bulgaria gelaufen, Bulgaria nach Serbia, Serbia nach Kroatia, Kroatia nach Slowenia, Slowenia nach Österreich, Österreich nach Deutschland. Alles gelaufen, komplett. Hat ein und halb Monate gedauert. Ob es regnet so Hagel, Flüsse reingehen, Meer reingehen, keine Ahnung, alles laufen. Kalt, warm, heiß, trotzdem mussten wir einfach weg. Und was anderes haben wir bezahlt. Mein Vater hat $9500 €$ bezahlt, nur für mich selber. Er hat, ich hab gesagt, wir haben Häuser gehabt, wir hatten viel in Irak gehabt. Der hat verkauft. Wir hatten zwei Wohnungen gehabt. Alles verkauft. (Interview 1, S. 15/Z. 21-28).

Dieser Ausschnitt aus dem Interview zeigt exemplarisch, was es bedeutet auf der Flucht zu sein und welche Fülle an körperlichen, psychischen und materiellen Ressourcen Geflüchtete investiert haben, um nach Deutschland zu gelangen. Auch wenn es vielen persönlich nicht möglich war, im Kontext der Interviews über ihre Flucht $\mathrm{zu}$ sprechen, wird aus den verbleibenden Interviewpassagen deutlich, welche Strapazen, Gewaltwiderfahrnisse und psychische Belastungen mit der Flucht verbunden waren. Die materielle Existenz musste oft zurückgelassen oder in die Flucht investiert werden. Gemeinsam ist allen Interviewten, dass sie aus Angst um ihr Leben und das ihrer Familienangehörigen geflohen sind und sich für Deutschland ein Leben in Sicherheit erhoffen. Die Flucht war eine aktive Form der Problemlösung. Die Angst 


\begin{tabular}{l|l|lll} 
& Männer/Jungen $(\boldsymbol{n})$ & Frauen/Mädchen $(\boldsymbol{n})$ & Interviews $(\boldsymbol{n})$ & Herkunftsländer \\
\hline Gemeinschaftsunterkünfte & 9 & 9 & 10 & Afghanistan, Syrien, Somalia \\
Eigene Wohnungen & 7 & 5 & 3 & Afghanistan, Syrien, Irak
\end{tabular}

im Herkunftsland war größer als die Angst vor der Flucht. Wohlbefinden ist für sie zuallererst mit dem Gefühl von Sicherheit verbunden.

In Deutschland angekommen sind Geflüchtete einerseits stark auf ehrenamtliche und professionelle Unterstützung angewiesen, um mit den Anforderungen des Status als Geflüchtete im Aufnahmeland zurechtzukommen, ihren Alltag zu gestalten und Sprachbarrieren zu überwinden. Andererseits entwickeln sie auch hier aktiv Handlungsstrategien, um mit den Herausforderungen des Alltags zurechtzukommen und ihren persönlichen Idealvorstellungen und Wünschen ein Stück näher zu kommen. Das Leben in Deutschland wird mit Wünschen z.B. nach einem unbefristeten Aufenthaltsrecht, einem guten Job mit gutem Einkommen, der Fortsetzung der bisherigen Berufsbiographie, einer verbesserten Situation für die eigenen Kinder oder auch nur einem ruhigen Lebensabend verbunden.

Die relative und oft befristete $\mathrm{Si}$ cherheit des Lebens in Deutschland ist für Geflüchtete zugleich damit verbunden, ein selbstbestimmtes Leben weitgehend aufgeben zu müssen. Was sie in Deutschland tun können, ist von ihrem Aufenthaltsstatus, der Anerkennung ihrer Bildungs- und Berufsabschlüsse, den vorhandenen Sprachkenntnissen und der Chance auf eine eigene Wohnung und einen Arbeitsplatz abhängig. Leben als Geflüchtete kann aufgrund fehlender Anerkennung von Qualifikationen und rechtlicher Hindernisse mit einem erheblichen sozioökonomischen Statusverlust verbunden sein. So führt die fehlende Anerkennung beruflicher Kompetenzen zur faktischen Dequalifikation. Handlungskompetenz geht darüber verloren. Aus dieser Diskrepanz zwischen aktiver Problemlösung und faktischer Handlungsunfähigkeit entstehen erhebliche psychosoziale Belastungen.

In einem anderen Interview mit einem etwa 60 -jährigen Jesiden aus dem
Irak, der vor den Jihadisten des Islamischen Staates geflohen ist, wird beispielsweise der Wunsch nach einem ruhigen Lebensabend ohne Angst um das eigene Leben und v. a. das der Familienangehörigen besonders deutlich. Zur Verwirklichung dieses Wunsches war die erste Handlungsstrategie im Herkunftsland alles aufzugeben und zunächst den Söhnen zur Flucht zu verhelfen, um dann so bald wie möglich nachzukommen und den Rest der Familie zu holen. Angekommen in Deutschland entwickelt er Strategien, einen möglichst unbefristeten Aufenthaltsstatus zu erlangen, Deutsch zu lernen, eine eigene Wohnung mit seiner Frau und mehreren Kindern zu erhalten. Bislang scheiterte er aber an den Wünschen, die traumatischen Erfahrungen aus dem Herkunftsland und der Flucht aufzuarbeiten, als Bauer wieder zu arbeiten, soweit sein Gesundheitszustand dies zulässt, mit dem verdienten Geld ein eigenes Haus zu bauen und der Familie eine neue Zukunft aufzubauen. In der Überwindung der vielen Hürden haben seine Kinder eine wichtige Funktion, denn nur durch diese besteht überhaupt die Möglichkeit, eines der Ziele zu erreichen. Für sie wünscht er sich, dass das Leben mehr Chancen bietet, als für ihn selbst.

Geflüchtete können demnach bei der Verwirklichung ihrer Ziele an unlösbare Schwierigkeiten geraten und sich als handlungsunfähig erleben. Es sind zugleich Menschen, die viele Ressourcen investiert haben, um nach Deutschland zu gelangen und z. T. eine Fülle von Ressourcen investieren, um ihrem Ziel der Selbstbestimmung über ihr Leben ein Stück näher zu kommen.

\section{Soziales Wohlbefinden und Integration}

Es gibt andere Leute, die wirklich keine Probleme haben, die von ihrer Heimat rausgegangen sind, erzählen vier, fünf Lügen, kriegen drei Jahre und er hat wirklich Probleme, Drohungen et cetera (..) wird grad mal ein Jahr bekommen oder geduldet sag ich mal. Am Anfang hatten die Duldung, da haben wirs weg gemacht durch Anwalt, dann haben die jetzt diese ein Jahr bekommen. So und das wünscht er sich halt, dass es in Regierung es sich halt was verändert, dass sie ernstgenommen werden und die Leute die verarschen eins auf den Deckel bekommen (...). (Interview 3, S. 15/Z. 3-9).

In diesem Interviewausschnitt wird deutlich, dass Geflüchtete bei der Ankunft in Deutschland damit konfrontiert werden, dass sie den Grund für ihre Flucht zunächst glaubhaft machen müssen, um als Flüchtlinge anerkannt zu werden oder zumindest subsidiären Schutz zu erhalten. Dafür fehlen ihnen oft Beweise. Nicht immer können sie nachvollziehen, weshalb ihnen nicht geglaubt wird und wieso sie beweisen müssen, dass die Flucht beispielsweise aus Afghanistan gerechtfertigt ist. Strukturell begegnet ihnen damit eine Kultur des Misstrauens, die sie nicht generell bei allen anderen für unbegründet halten, aber jeweils in ihrem persönlichen Fall. Unabhängig vom Asylverfahren setzt sich diese Kultur des Misstrauens in den Erfahrungen in ihrem Alltag fort, wenn sie aufgrund kultureller Unterschiede auf fehlende Akzeptanz stoßen. Sie fühlen sich nicht ernst genommen, isoliert, diskriminiert und nicht gleichbehandelt. Sie beklagen, kaum Kontakt zu Deutschen zu bekommen, weder in den Gemeinschaftsunterkünften noch in eigenen Wohnungen. Ihnen fehlen Möglichkeiten der Integration, die ihnen zugleich die Chance bieten könnten, ihre Sprachkompetenzen alltagsnah zu verbessern. Sie fühlen sich ausgegrenzt. Dies steht im Kontrast dazu, dass ihnen unterstellt wird, sich in Deutschland nicht integrieren zu wollen.

So berichtet eine syrische Familie mit guten Deutschkenntnissen beispielsweise, dass sie fast nur Kontakt zu anderen syrischen Familien haben. Lediglich ein ehrenamtlicher Helfer kommt sie besu- 


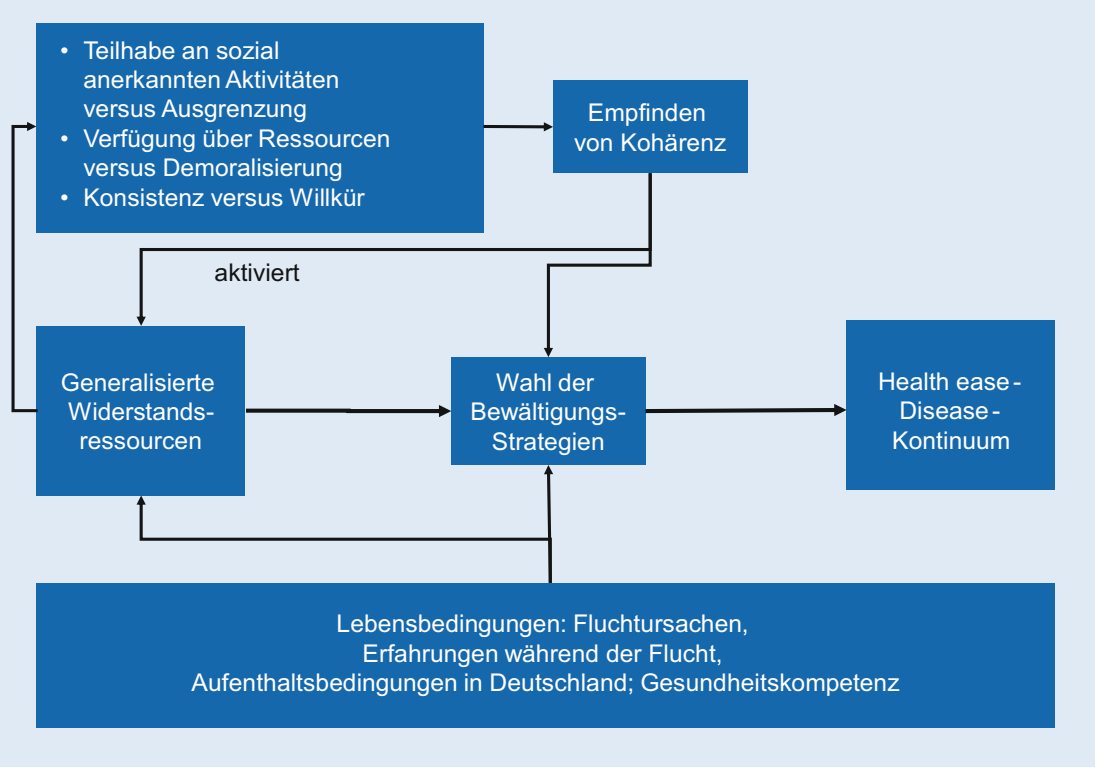

Abb. 2 \ Vereinfachtes Modell der Entstehung von Gesundheit bei Geflüchteten in Anlehnung an die Theorie der Salutogenese von Aaron Antonovsky [1]

chen und spielt mit den Kindern. Trotz guter Qualifikationen ist die Integration von Vater und Bruder in den Arbeitsmarkt bislang nicht gelungen. Die Mutter nimmt an Nähkursen teil, in denen sie aber nur Geflüchtete trifft.

Die Anforderungen an und die Chancen auf Integration scheinen aus Sicht der Geflüchteten im Kontrast zueinander zu stehen. Ihnen kann das Gefühl der Teilhabe an sozial anerkannten Aktivitäten sowie das Gefühl von Akzeptanz und Gleichbehandlung fehlen und damit eine zentrale Gesundheitsressource.

\section{Gesundheitsbeeinträchtigungen}

Wir sind immer mit unseren Eltern. So wenn gibt es ein etwas wo trennt uns von unsere Eltern, das macht uns sehr (..) vielleicht krank. Das ist was ich denke. Das macht meine Erkrankung schlechter. Meine Multiple Sklerose ist schlechter geworden. Ich muss ein höheres Medikamentenmodell nehmen, weil meine Erkrankung ist schlechter jetzt, als ich in Syrien war. (Interview 2, S. 14/Z. 10-14).

Bei den interviewten Geflüchteten waren schwerwiegende chronische Erkrankungen oder körperliche Beeinträchtigungen bei ihnen oder ihren Kindern häufig, die sich durch die Gründe für die Flucht, die Flucht oder das Leben in Deutschland teilweise verschlechtert haben. Im Datenmaterial ließ sich kein Beispiel für eine Verbesserung der gesundheitlichen Versorgung finden, obwohl alle Interviewten die gute Gesundheitsversorgung in Deutschland loben. Der Interviewausschnitt zeigt exemplarisch, dass die Gründe für die Verschlechterung oft mit den Bedingungen der Flucht und dem Leben als Geflüchtete in Verbindung gebracht werden.

Geflüchtete nehmen zugleich war, dass sie nicht in der gleichen Art und Weise an der guten Gesundheitsversorgung in Deutschland partizipieren können. Hinderungsgründe sind der Status als Geflüchtete sowie Sprachprobleme, die es ihnen nicht ermöglichen, störungsfrei mit dem Gesundheitspersonal zu kommunizieren. Damit bleiben auch Chancen ungenutzt, Handlungskompetenzen zum Erhalt der eigenen Gesundheit zu entwickeln und auszubauen.

So berichtet ein Afghane beispielsweise, dass bei ihm zwei Augenoperationen notwendig sind, die erst dann durchgeführt werden können, wenn ihm zumindest subsidiärer Schutz zugesprochen wird. Auch aufgrund seiner Augenprobleme spricht er kaum Deutsch. So sitzt er viel zu Hause und raucht aus
Langeweile. Die Mutter eines behinderten Kindes kann an Integrationsangeboten nicht teilnehmen, weil sie sich ununterbrochen um ihr Kind kümmern muss. Insofern hat sie auch keine Chance, eigene Gesundheitspotentiale zu entwickeln. Eine junge Frau ist nach einem Unfall in ihrem Herkunftsland in der Gemeinschaftsunterkunft auf einen Rollstuhl angewiesen, hat aber keine Person, die ihr bei der Alltagsbewältigung hilft.

Die Chancen, Gesundheitskompetenzen zu entwickeln, scheinen aus mehreren Gründen eingeschränkt zu sein: den Sprachproblemen, dem eingeschränkten Kontakt zur Gesundheitsversorgung und den Lebensbedingungen, die es erschweren, einen gesundheitsfördernden Lebensstil umzusetzen.

\section{Diskussion}

Im Rahmen der Studie ist es nicht möglich geworden, eine theoretische Sättigung der Daten zu erreichen. Vor allem hat sich die Entscheidung zur Durchführung von Familieninterviews, das Angewiesensein auf männliche Sprachmittler und die Anwesenheit auch von männlichen Interviewern bei den Interviews mit Frauen nachteilig auf die Chancen ausgewirkt, konkrete Sichtweisen von geflüchteten Frauen zu erheben. Die Frauen haben sich an den Gesprächen oft als mehr oder weniger stumme Zuhörerinnen beteiligt, in den Einzelgesprächen sehr deutlich gemacht, dass sie über traumatisierende Erfahrungen auf der Flucht oder in Deutschland nicht sprechen möchten oder die Söhne und Sprachmittler haben für sie aufgrund fehlender Sprachkenntnisse gesprochen. Es waren eher die Männer und die Söhne, die das Gespräch in den Familien bestritten haben.

Auch könnte die Anwesenheit einer Kontaktperson von einer Wohlfahrtsorganisation sowohl förderlich als auch hinderlich für die Bereitschaft offen zu sprechen gewesen sein. Zudem stellte es für die Interpretation ein Hindernis dar, dass nur auf den ins Deutsche übersetzen Text zurückgegriffen werden konnte. Insofern geben die analysierten Daten lediglich Hinweise, in welche Richtung weiter geforscht werden muss. Eine genauere Ana- 
lyse der Sicht insbesondere von geflüchteten Frauen ist zwingend notwendig.

Die analysierten Daten lassen allerdings die Schlussfolgerungen zu, dass die Rahmenbedingungen des Lebens als Geflüchtete in Deutschland einerseits nicht hinreichend zu einer Verbesserung von Gesundheitskompetenz beitragen, andererseits weitergehend zentrale Gesundheitsressourcen eher verloren gehen können. Statt in einer Situation des Mangels eigene Stärken und Ressourcen entdecken und entwickeln zu können, können die Bedingungen des Lebens als Geflüchtete in Deutschland eher zur Demoralisierung [9] führen, dem Gegenpol des Empfindens von Kohärenz [1]. Dies ist verbunden mit dem Verlust von Selbstbestimmung und der mangelnden Erfahrung der Teilhabe an sozial anerkannten Aktivitäten in Deutschland, also der Ausgrenzung, sowie ggf. mit dem Empfinden von Willkür bei Asylentscheidungen oder einer Zubilligung medizinischer Versorgung.

Die - Abb. 2 zeigt in vereinfachter Form, wie das Modell der Salutogenese auf die Lebensrealität von Geflüchteten bezogen werden kann. Der Theorie der Salutogenese [1] zu Folge sind es die Lebenserfahrungen der Teilhabe, der Verfügung über geeignete Ressourcen zur Bewältigung der Anforderungen und der Konsistenz, die das Empfinden von Kohärenz prägen. Ihr Gegenstück ist die Ausgrenzung, die Demoralisierung und das Empfinden von Willkür. Das Kohärenzempfinden entscheidet darüber, welche generalisierten Widerstandsressourcen aktiviert werden und in welcher Vielfalt Bewältigungsstrategien gewählt werden. Es könnte angenommen werden, dass Geflüchtete über ein hohes Empfinden von Kohärenz verfügen müssen, um dem Weg nach Deutschland zu schaffen. Zugleich sind die Ursachen der Flucht, die Erfahrungen auf der Flucht und die Erfahrungen in Deutschland Lebenserfahrungen, die genau dieses Empfinden beeinträchtigen könnten. Genau hier muss die Gesundheitsförderung mit Geflüchteten ansetzen: Lebenserfahrungen der Teilhabe an sozial anerkannten Aktivitäten fördern. Damit dies nicht zu einer Sisyphusarbeit wird, scheint ein Healthin-all-policies-Ansatz unvermeidlich.

\section{Fazit für die Praxis}

- Über Sichtweisen von Geflüchteten zu ihren Bedürfnissen bedarf es weiterer Forschung, die die Perspektive von Männern und Frauen getrennt erforschen muss.

- Gesundheitsförderung mit Geflüchteten muss sich primär darauf konzentrieren, Lebenserfahrungen in Deutschland zu unterstützen, die das Empfinden von Kohärenz stabilisieren.

- Gesundheitsförderung mit Geflüchteten bedarf einer öffentlichen Finanzierung, da es sich um eine gesundheitlich benachteiligte Adressatengruppe handelt.

\begin{tabular}{l} 
Korrespondenzadresse \\
$\begin{array}{l}\text { Stechschie Karg } \\
\text { Leipziger Straße 123, } \\
\text { 36037 Fulda, Deutschland } \\
\text { stephanie.karg@ } \\
\text { pg.hs-fulda.de }\end{array}$ \\
\hline
\end{tabular}

Danksagung. Die Autor*innen bedanken sich bei Lisa Rose, Freya Bongard, Antonia Abwander, Jasmin Helbach, Oliver Mayer, Sebastian Stapfer, Johannes Wiegand, Leona Aschentrup, Tatjana Hermann und Maren Kubandt für die Mitarbeit im Projekt.

Förderung. Das Projekt wurde aus hochschulinternen Mitteln gefördert.

Funding. Open Access funding provided by Projekt DEAL.

\section{Einhaltung ethischer Richtlinien}

Interessenkonflikt. S. Karg, B. Blättner und T. Süleyman Igde geben an, dass kein Interessenkonflikt besteht.

Von allen Interviewpartnern liegt eine Einverständniserklärung zur Datenerhebung vor, die auf Arabisch übersetzt wurde. Datenschutzbestimmungen wurden durchweg eingehalten; ethische Standards orientierten sich an den Vorgaben der zuständigen Ethikkommission.

Open Access. Dieser Artikel wird unter der Creative Commons Namensnennung 4.0 International Lizenz veröffentlicht, welche die Nutzung, Vervielfältigung, Bearbeitung, Verbreitung und Wiedergabe in jeglichem Medium und Format erlaubt, sofern Sie den/die ursprünglichen Autor(en) und die Quelle ordnungsgemäß nennen, einen Link zur Creative Commons Lizenz beifügen und angeben, ob Änderungen vorgenommen wurden.
Die in diesem Artikel enthaltenen Bilder und sonstiges Drittmaterial unterliegen ebenfalls der genannten Creative Commons Lizenz, sofern sich aus der Abbildungslegende nichts anderes ergibt. Sofern das betreffende Material nicht unter der genannten Creative Commons Lizenz steht und die betreffende Handlung nicht nach gesetzlichen Vorschriften erlaubt ist, ist für die oben aufgeführten Weiterverwendungen des $\mathrm{Ma}$ terials die Einwilligung des jeweiligen Rechteinhabers einzuholen.

Weitere Details zur Lizenz entnehmen Sie bitte der Lizenzinformation auf http://creativecommons.org/ licenses/by/4.0/deed.de.

\section{Literatur}

1. Antonovsky A (1997) Salutogenese. Zur Entmystifizierung der Gesundheit. dgvt, Tübingen

2. BAMF - Bundesamt für Migration und Flüchtlinge (2014) Das deutsche Asylverfahren - ausführlich erklärt. Zuständigkeiten, Verfahren, Statistiken, Rechtsfolgen. https://www.bamf.de/DE/Themen/ AsylFluechtlingsschutz/AblaufAsylverfahrens/ ablaufasylverfahrens-node.html. Zugegriffen: 13. Apr. 2020

3. BAMF - Bundesamt für Migration und Flüchtlinge (2020) Aktuelle Zahlen. Ausgabe Januar 2020. https://www.bamf.de/SharedDocs/Anlagen/DE/ Statistik/AsylinZahlen/aktuelle-zahlen-januar2020.pdf?_blob=publicationFile \&v=3. Zugegriffen: 12. Apr. 2020

4. Dahlgren G, Whitehead M (1991) Policies and strategies to promote social equity in health. Institute for Futures Studies, Stockholm

5. Dresing T, Pehl T (2015) Praxisbuch Interview, Transkription \& Analyse. Anleitungen und Regelsysteme für qualitativ Forschende, 6. Aufl. Eigenverlag, Marburg

6. Fegert JM, Diehl C, Leyendecker B, Hahlweg $\mathrm{K}_{\text {, }}$ Wissenschaftlicher Beirat (2017) Aus Kriegsgebieten geflüchtete Familien und ihre Kinder: Entwicklungsrisiken, Behandlungsangebote, Versorgungsdefizite. Bundesministerium für Familie, Senioren, Frauen und Jugend, Berlin (Kurzgutachten des Wissenschaftlichen Beirats für Familienfragen)

7. Helfferich C (2011) Die Qualität qualitativer Daten. Manual für die Durchführung qualitativer Interviews, 4. Aufl. VS, Wiesbaden

8. Kaba-Schönstein L (2018) Gesundheitsförderung 1: Grundlagen. BZgA (Hsg.): Leitbegriffe der Gesundheitsförderung. https://www. leitbegriffe.bzga.de/alphabetisches-verzeichnis/ gesundheitsfoerderung-1-grundlagen/. Zugegriffen: 12. Apr. 2020

9. Keupp H (2006) Gesundheitsförderung als Identitätsarbeit. Z Qual Bild Berat Sozforsch 7(2):217-238 (https://nbn-resolving.org/urn:nbn: de:0168-ssoar-277954)

10. Strauss A (1991) Grundlagen qualitativer Sozialforschung. Datenanalyse und Theoriebildung in der empirischen soziologischen Forschung. Fink, München

11. UNHCR - United Nations High Commissioner for Refugees (2019) Global trends. Forced displacement in 2018. https://www.unhcr.org/dach/de/ services/statistiken.Zugegriffen: 12. Apr. 2020 